\title{
SPICULES ARE BRIGHT AND DARK
}

\author{
HAROLD ZIRIN \\ Big Bear Solar Observatory, Hale Observatories, Carnegie Institution of Washington, \\ California Institute of Technology, Pasadena, Calif., U.S.A.
}

\begin{abstract}
I have tried to understand whether spicules appear dark or bright against the disk in $\mathrm{H} \alpha$ by comparing high resolution pictures in $\mathrm{H} \alpha$ and $\mathrm{Ca}$ II K. Since the chromospheric structure is more apparent near active regions, I have first compared the $\mathrm{H} \alpha$ and $\mathrm{K}$ fibrils near an active sunspot; these are illustrated in my review paper (this Symposium, p. 161). The elongated fibrils in the active center appear similar to spicules, but much larger and more horizontal. $\mathrm{K}$ line pictures show bright features better, while $\mathrm{H} \alpha$ shows dark features better.

Careful comparison of the $\mathrm{K}$ and $\mathrm{H} \alpha$ pictures shows a complete correspondence. Bright fibrils are bright in both lines and dark features, dark. The only difference is in the relative contrast. As one can judge from these pictures, there seem to be an equal number of bright and dark fibrils in the active region, the former being more prominent on the $\mathrm{K}$-line flares and the latter, on the $\mathrm{H} \alpha$. It is not surprising that this agreement exists. Both bright and dark fibrils seem to emanate from bright plages or plagettes.

In Figure 1, we see an $H \alpha: K$ pair taken near the limb. In this case the $\mathrm{K}$-line frame is unfortunately taken with $0.6 \AA$ bandpass, which penetrates a little lower into the chromosphere, and does not show spicules very well. We have marked several pairs of bushes by corresponding numbers. The following points appear:
\end{abstract}

(1) The bases of the bushes show bright vertical structure in $\mathrm{H} \alpha$ (bush 2 is typical) with dark spicules above. However, there is not a clear correspondence between the bright jet at the base and the vertical absorption above.

(2) The bright bases in $\mathrm{K}$ correspond to the bright bases in $\mathrm{H} \alpha$, but near the limb $(3,4,5)$ much more vertical bright structure appears in $\mathrm{K}$, partly because of the lower absorption by foreground objects.

(3) Some bright $\mathrm{H} \alpha$ spicules are seen, for example in the bushes 6 and 7, but they are in general few.

Thus the bush structure near the limb in quiet regions is different from that in active regions; there is a clear bright-dark asymmetry, and only a few spicules are hot enough or optically deep enough to go into emission in $\mathrm{H} \alpha$.

\section{DISCUSSION}

Athay: I would like to make one comment, but I don't mean it to be as bad as it sounds. I did not see one bright mottle that you convinced me was a spicule.

Zirin: You're the one guy that escaped seeing the enlargements so far.

Wilson: You must have a certain amount of faith to see spicules - if Hal says he sees spicules I'll believe him. 


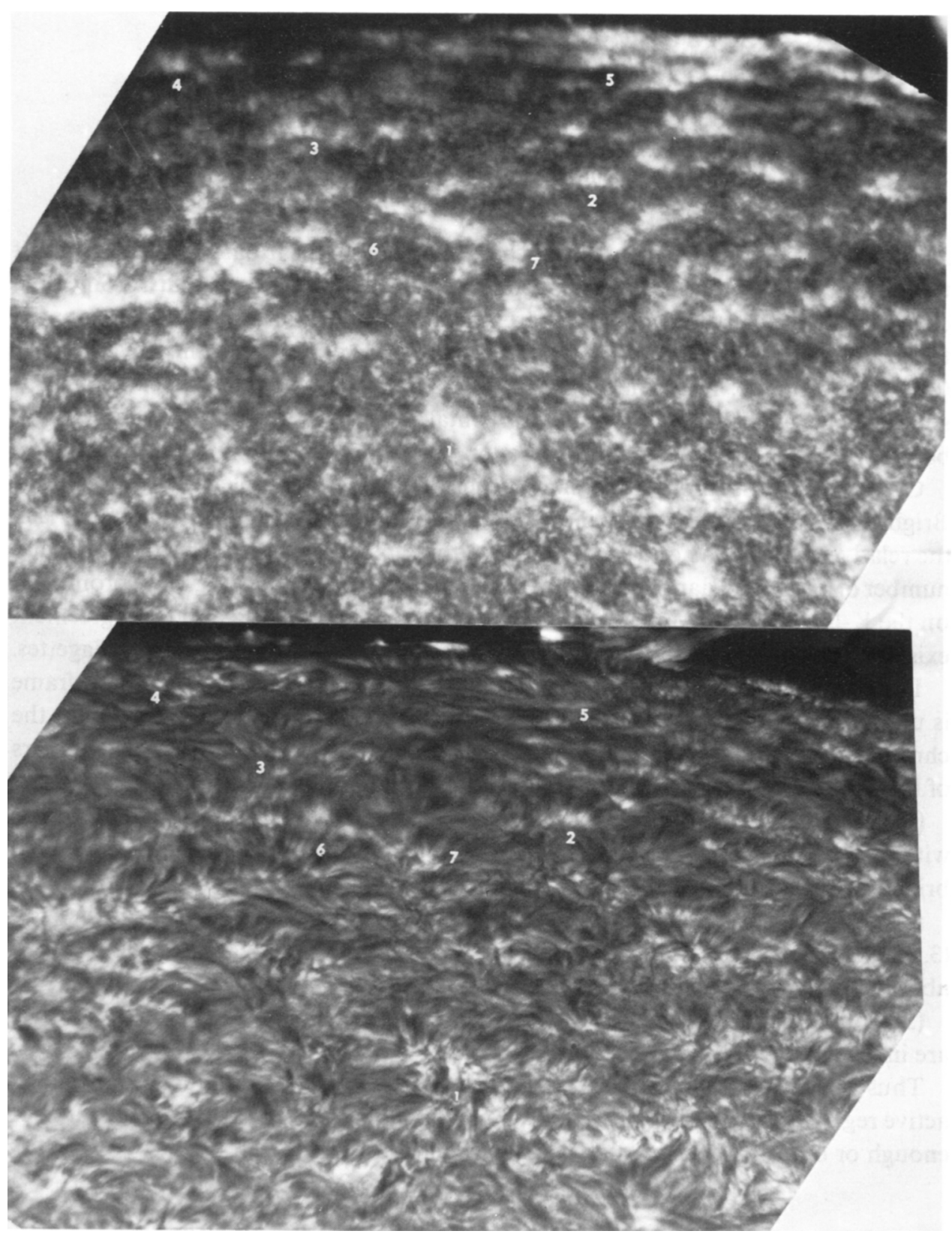

Fig. 1. Filtergrams near the limb taken 1971 , Sept. 23. K line $0.6 \AA$ wide, above, $\mathrm{H} \alpha, 0.25 \AA$ wide, below. The granular $\mathrm{K}$-line structure inside the network cells corresponds to a lower height. These pictures were made $5 \mathrm{~s}$ apart. 